\title{
MULTIMEDIA E INTERACTIVIDAD EN EL MATERIAL DOCENTE DE SOPORTE Y SU APLICACIÓN A LOS ESTUDIOS DE COMUNICACIÓN ${ }^{1}$
}

\author{
Xavier Ribes; xavier.ribes@uab.cat \\ Montse Bonet; montserrat.bonet@uab.cat \\ Josep Àngel Guimerà i Orts; josepangel.quimera@uab.cat \\ David Fernández-Quijada; david.fernandez@uab.cat \\ Luísa Martínez-García ; luisa.martinez@uab.cat \\ Departament de Comunicació Audiovisual i de Publicitat. \\ Universitat Autònoma de Barcelona
}

\section{RESUMEN}

El artículo presenta los principales resultados de una encuesta realizada a docentes y discentes de la Facultad de Ciencias de la Comunicación de la Universitat Autònoma de Barcelona sobre una muestra de material docente de apoyo, para cuya elaboración se utilizó el programa informático Camtasia Studio. Los resultados muestran actitudes positivas hacia el uso de este tipo de materiales aunque con percepciones diferenciadas por parte de cada colectivo.

PALABRAS CLAVE: material docente, Camtasia Studio, estudios de comunicación

\section{ABSTRACT}

This paper shows the main results of a survey answered by teachers and students in the Faculty of Communication Sciences at the Universitat Autònoma de Barcelona about a sample of supporting learning content, made with Camtasia Studio software. The results show a positive attitude towards the use of these materials although some differences can be found between teachers and students.

KEY WORDS: supporting learning content, Camtasia Studio, Communication studies

\footnotetext{
${ }^{1}$ Este artículo forma parte del proyecto de mejora de la calidad docente Recursos docents digitals elaborats amb Camtasia Studio per als estudis de la Facultat de Ciències de la Comunicació (2008MQD 00117), financiado por la Agència de Gestió d'Ajuts Universitaris i de Recerca (AGAUR) de la Generalitat de Catalunya.
} 


\section{VIEJAS NECESIDADES, NUEVOS RECURSOS ${ }^{2}$}

La necesidad de adecuar la metodología docente al siglo XXI no es ni nueva ni exclusiva del Espacio Europeo de Educación Superior, aunque en ocasiones así pueda deducirse. Hubo ya un primer cambio importante a principios de los años 90 (cuando nacieron los estudios que precisamente pronto van a ser sustituidos). Entonces se adaptó la metodología docente, ajustándose más a los nuevos perfiles precisados por la industria, sin renunciar por ello a la crítica y a la reflexión (Bonet, Martí y Pérez-Portabella, 2005): se introdujeron más asignaturas específicas; aumentó la carga docente práctica y aplicada (grado de experimentalidad); se apostó por el trabajo en equipo reproduciendo al máximo las rutinas productivas de la industria al tiempo que se construía colectivamente el conocimiento; y se dio mayor protagonismo a las tecnologías. La evolución de los estudios de comunicación son un excelente observatorio para conocer la propia evolución de las industrias culturales españolas y el contexto mediático en general (Bonet, 2006).

Además, los estudios de comunicación guardan una estrecha relación con las TIC (tecnologías de la información y la comunicación): como instrumento reproductor, en las clases prácticas, de las rutinas productivas; como objeto de estudio, dentro y fuera de las aulas; aplicándose a la docencia (Bonet, 2006). Cuando gracias a la aplicación de las TIC se pasa de centrar la atención sobre el que enseña a centrarla sobre el que aprende, pasamos también del trabajo individual al trabajo en red y en grupo, de un profesor a la defensiva a uno responsable, de proveer información a favorecer un aprendizaje activo (Harvey y Knight, citados en Aiello y Willem, 2004).

Sin duda, las TIC transforman el contexto educativo así como la cantidad y la calidad de la información a la que accedemos; el modo en que se codifica esta información y el modo en que accedemos a ella (Grané, 2004).

En un intento de ajustar una vez más la docencia a las nuevas exigencias metodológicas, sin obviar las características propias de los estudios de comunicación, un grupo de profesores de la Facultad de Ciencias de la Comunicación de la Universitat Autònoma de Barcelona (UAB) llevaron a cabo la primera fase de un estudio exploratorio (que se quiere más ambicioso en el tiempo) para validar el material docente digital de soporte a la docencia que ellos mismos diseñaron, utilizando para ello el programa Camtasia Studio. En la base está la voluntad de elaborar un protocolo de guía para cualquier docente que desee producir material de soporte para sus materias así como crear un repositorio con ese material. Dada la complejidad de ambos objetivos, se dividió el estudio en dos fases, siendo la primera la que se describe aquí. Por lo tanto, en esta primera etapa se diseñó y produjo una muestra de material digital de soporte, se diseñaron las encuestas que deberían someter a prueba el prototipo producido, se sometió a test la muestra elaborada (sobre diapositivas complejas de PowerPoint, más adelante se pretende trabajar con tutoriales de editores de vídeo y audio) y se analizaron los resultados obtenidos de las encuestas, realizadas tanto a docentes como a discentes. Dichos resultados deberán servir para afrontar la segunda etapa, modificar el prototipo, realizar entrevistas en profundidad y elaborar el protocolo.

\footnotetext{
${ }^{2}$ Work in progress: una primera versión de este estudio se presentó en julio de 2008 en el Congreso Internacional de Docencia Universitaria e Innovación (CIDUI). Con posterioridad, se explotaron los resultados de la segunda parte de las encuestas, incluidos ahora en este trabajo. Actualmente, el equipo se halla en proceso de preparación de la segunda fase del proyecto.
} 


\section{EL MATERIAL DIDÁCTICO Y LA HERRAMIENTA: ¿POR QUÉ CAMTASIA STUDIO?}

Camtasia Studio, de la compañía Techsmith, es un programa que permite grabar como secuencia de vídeo todas las acciones que aparecen en la pantalla del ordenador, sin importar su fuente original, por lo cual realiza una aproximación multimedia realmente enriquecedora pues combina vídeo, audio, texto, gráficos, animaciones, etc. (aunque sólo funciona con el sistema operativo Windows). Con este programa se puede grabar, editar, añadir audio, crear menús personalizados, incorporar iconos, flechas, etc. y trabajar con una interfaz de línea de tiempo, al estilo de los programas de edición de audio y vídeo utilizados en los estudios de comunicación y entornos profesionales, como Final Cut Pro, Adobe Flash o las diferentes soluciones de la familia Avid, siendo por ello que resulta una interfaz familiar para muchos docentes de la facultad. Al mismo tiempo, la creación de productos multimedia forma parte también de la propia docencia y es por este motivo que una parte importante del profesorado conoce estos sistemas y sus exigencias en cuanto a destrezas, aunque en las encuestas realizadas a los profesores en este estudio, sólo un $10 \%$ demostró saber qué es este programa en concreto.

Dos de las mayores ventajas de Camtasia Studio es que el resultado puede exportarse en diversos formatos y que, además, dispone de otras funcionalidades como por ejemplo la posibilidad de insertar pantallas con preguntas dentro de la línea de tiempo, por lo que si el usuario no las responde adecuadamente la presentación no avanza. Este recurso es una herramienta de enormes potencialidades que aporta interactividad y facilita el proceso de autoevaluación.

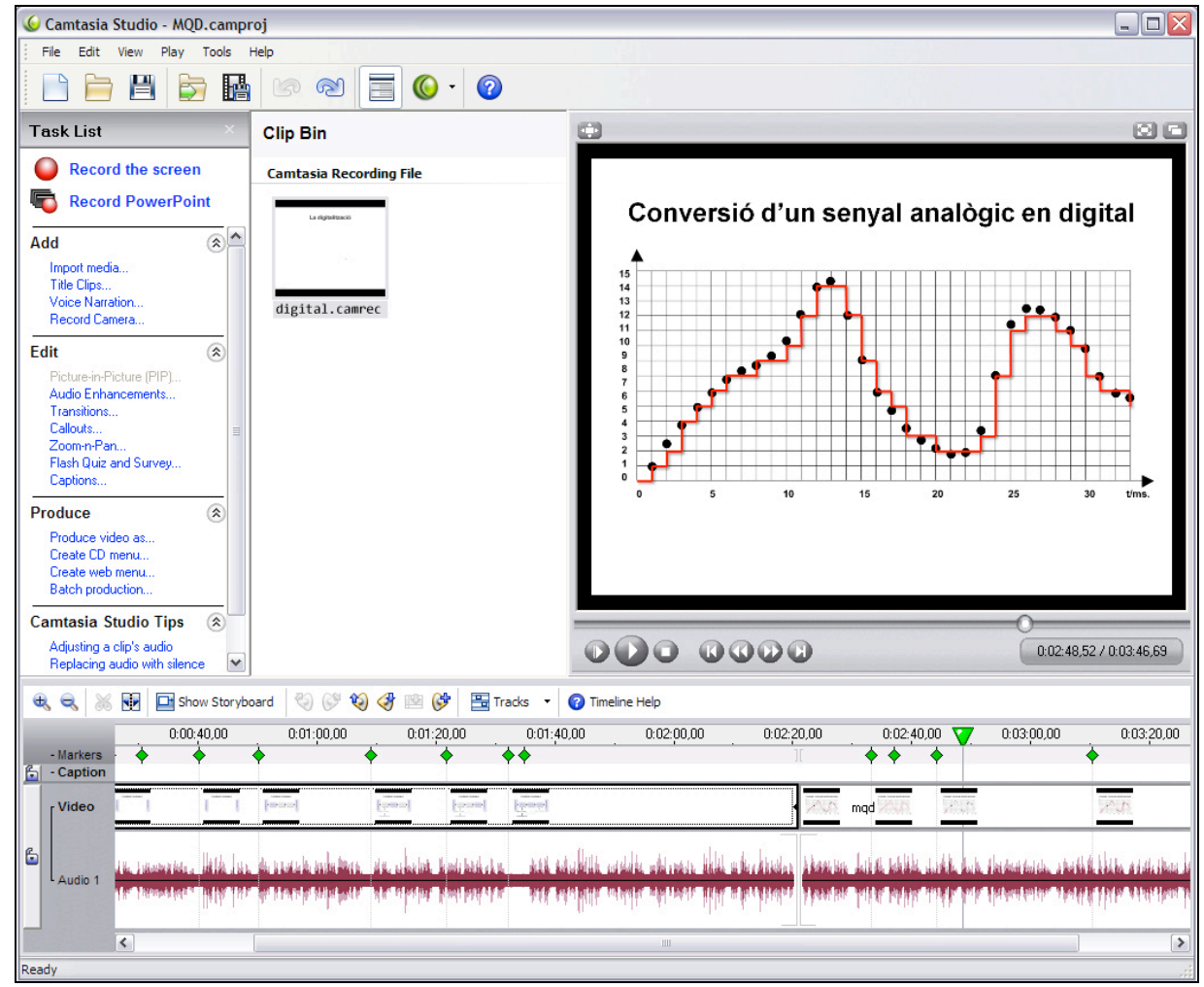

Imagen 1. Interfaz de trabajo de Camtasia Studio Fuente: elaboración propia. 


\section{EL MATERIAL SOMETIDO A PRUEBA: METODOLOGÍA, MUESTRA Y RESULTADOS}

Este análisis se fundamenta en una serie de preguntas, dudas y experiencias docentes que sirvieron de motivación para emprender este trabajo y que pueden resumirse de la siguiente forma:

- Los estudios de comunicación trabajan con muchos procesos y software y constantemente se necesitan tutoriales, guías y todo tipo de material de apoyo;

- Sin necesidad de esperar a la aparición del proceso de bolonia, la facultad de ciencias de la comunicación lleva tiempo realizando muchas clases a partir de trabajos en colaboración y con seguimiento tutorizado y ello requiere igualmente de material de calidad;

- Se constata que a menudo una parte del profesorado es reacio a las nuevas tecnologías y más todavía si se trata de aplicarlo a la docencia, es decir, cuando no se trata de reproducir una rutina productiva, aunque no rechaza la validez del material de soporte;

- Dadas las características propias de los estudios de comunicación, una de las preguntas más evidentes era si resultaría igualmente válido diseñar material de este tipo para las clases teóricas;

- Se partía de la base de un convencimiento bastante sólido de que este tipo de material sería muy bien aceptado, sobre todo por los alumnos y que entre los profesores habría opiniones más diversas;

- Se pretendía buscar una herramienta y preparar un protocolo que proporcionara autonomía al profesorado, tanto si diseñaba el material como si lo encargaba (pues tenía que saber qué ofrece la herramienta y qué debía pedir);

- Se tenía también el convencimiento de que la incorporación de material digital de soporte a la docencia conduciría lentamente a un cambio en el proceso de enseñanza-aprendizaje (E-A).

El primer paso fue elaborar una animación para ser sometida a prueba pero para ello era necesario decidir cuál sería su contenido. El equipo mantuvo diversas reuniones con el fin de elegir el contenido más adecuado y éste se eligió pensando en qué material suelen compartir de forma obligada las tres licenciaturas propias del ámbito de la comunicación (Periodismo, Comunicación Audiovisual y Publicidad y Relaciones Públicas). Tras un repaso de las diversas materias ofertadas y elaborar una lista con algunos de los temas de mayor dificultad en su explicación, se optó finalmente por el estudio de la digitalización como proceso tecnológico básico y más por cuanto nos estamos refiriendo al ámbito de la comunicación y se imparten, de hecho, asignaturas como Tecnología de los Medios Audiovisuales. En definitiva, se tuvo en cuenta que la digitalización es un proceso de cierta complejidad (estamos hablando de alumnos cuyo objetivo académico primero no es ser ingenieros) y, por lo tanto, disponer de material de recordatorio y soporte podría ser valorado de forma positiva. Este material, debe quedar claro, se concibió para dar apoyo a la docencia, nunca como sustituto de las clases. 
El trabajo se repartió entre el equipo pues dos fueron los profesores encargados de elaborar el PowerPoint de base (a decir verdad, estaba ya preparado dado que se había utilizado en más de una clase) mientras que los otros empezaron la labor de preparación de las encuestas.

El resultado fue un vídeo de casi cuatro minutos de duración en el que se explica de forma general en qué consiste el proceso de digitalización. Cada una de las imágenes se acompaña de una voz en off explicativa. El vídeo puede pararse en cualquier momento y llevarse hacia delante o hacia atrás.

En cuanto a su duración, se tuvo en cuenta que al ser material de soporte ubicado en un archivo digital, sería preferible trabajar con piezas cortas y fácilmente consultables. Como nos recuerda O'Day (2006: 5), "Ias animaciones, al igual que otras herramientas de enseñanza, son más efectivas cuando el estudiante tiene un elemento de control. Muchos estudios avalan el concepto de que la interactividad refuerza el proceso de aprendizaje (Tversky and Morrison, 2002)". Esta interactividad, prosigue el autor, puede ser algo tan sencillo como reproducir, parar, rebobinar o volver a inciar una animación o bien puede tratarse de formas más complejas que obliguen al estudiante a responder correctamente una seria de preguntas antes de poder proseguir (de hecho, Camtasia Studio permite esta función, aunque todavía no se ha experimentado en su totalidad).

El uso de la voz no fue casual pues en más de una ocasión se discutió su necesidad y papel en la animación e incluso se habló de la posibilidad de incluir, en un estadio posterior, subtítulos para estudiantes con algún tipo de deficiencia auditiva. Finalmente, la opción de acompañar las imágenes con la voz se basó en la apuesta por la elaboración de un material multimedia que combinara distintas matrices.

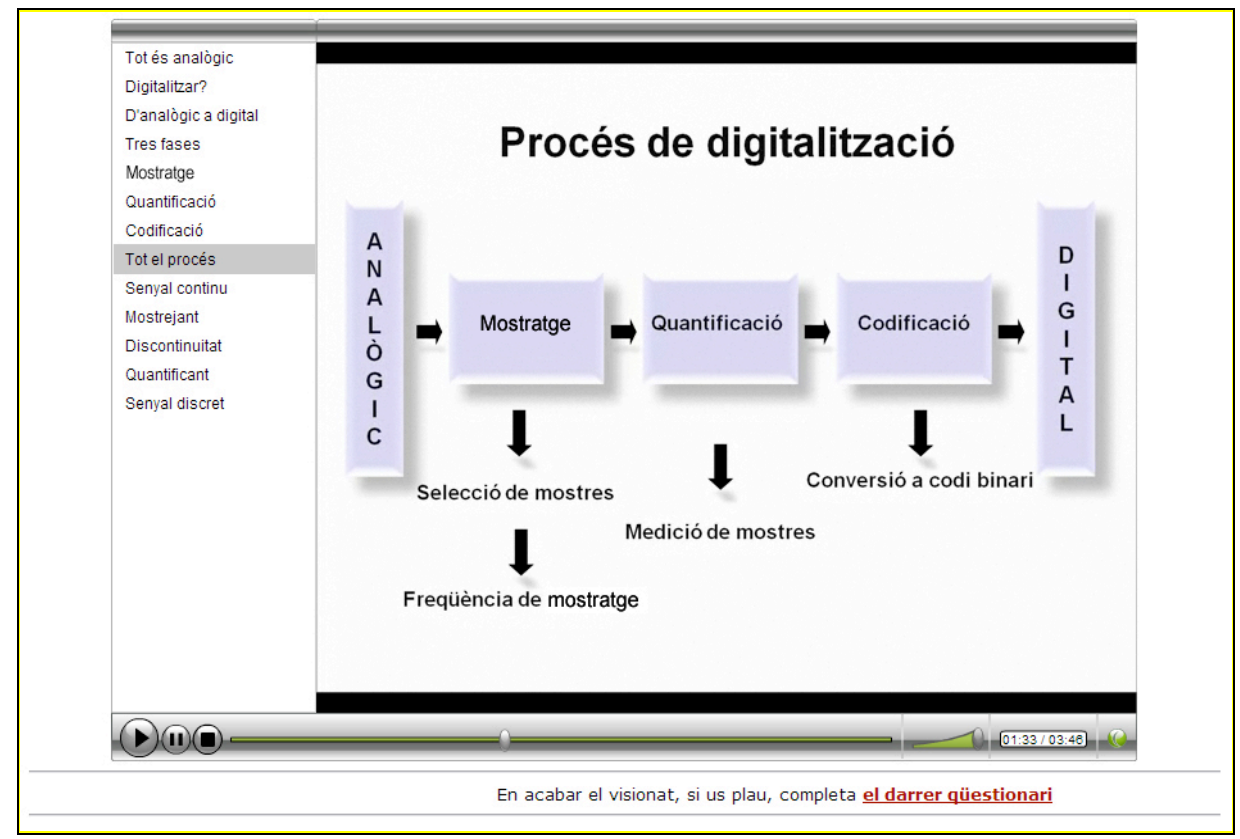

Imagen 2. Muestra de material docente elaborada con Camtasia Studio Fuente: elaboración propia.

Una vez elaborada la animación, el equipo procedió a visionarlo con el fin de controlar el máximo de detalles y someterlo a un primer examen. Posteriormente, se colgó en el servidor personal de 
uno de los profesores, a la espera de terminar el proceso metodológico de elaboración de las encuestas y poder hacer pública la dirección para su visionado. Precisamente, otro de los aspectos discutidos fue el de si la animación se pasaría un día y hora determinados en el que se hubiera convocado a cierta cantidad elegida de alumnos, reunidos todos en una sala (con ordenadores) o bien quedaría en línea para su consulta cuándo, dónde y cuantas veces quisiera cada alumno. Lo mismo se pensó para el caso de los profesores sometidos a encuesta. Finalmente, con total unanimidad, se optó por reproducir el mismo entorno de consulta que se prevé para cualquier material de este tipo, aun sabiendo que ello podía generar problemas de diversa índole como, por ejemplo, los referidos al ancho de banda y los formatos del vídeo.

Así, los cuestionarios estuvieron on-line puesto que se preveía que el uso y el archivo del material docente se haría mediante esta plataforma y se intentó que los encuestados hicieran el test en una situación lo más parecida posible a cómo la utilizarían normalmente, en caso de existir un catálogo con material de este tipo. Así fue como docentes y discentes visionaron la animación y respondieron el cuestionario. El único límite establecido fue el de las fechas en las que debían hacerlo, para lo cual se dejó un margen de 15 días (pensando, entre otros motivos, en el fin del primer semestre y el inicio de los exámenes).

En esta primera etapa, el diseño metodológico era de matriz cuantitativa, de tal forma que se prepararon dos encuestas con cuestionarios mixtos (preguntas cerradas y abiertas), una dirigida a los docentes y otra, a los discentes, aunque ambas compartían la misma estructura: un primer bloque, previo al visionado, con preguntas sobre las habilidades de los sujetos estudiados respecto al uso y el conocimiento de las nuevas tecnologías; después, el visionado del producto multimedia interactivo en sí y, finalmente, el segundo bloque de preguntas sobre el producto recién visionado. Sólo este segundo bloque de preguntas presentaba ciertas diferencias entre ambos colectivos.

La muestra de estudiantes y de profesores se obtuvo a partir de un diseño estratégico aplicado en dos etapas (Soriano, 2007): elección de una muestra de asignaturas ${ }^{3}$ basándose en que fueran troncales $u$ obligatorias a poder ser comunes a las tres licenciaturas, que hubiera materias que combinaran una elevada carga práctica y otras que fueran fundamentalmente teóricas, y que las hubiera de los cuatro cursos. Por tanto, los casos considerados no son representativos del universo desde el punto de vista estadístico.

La muestra de profesores, elaborada a partir de las asignaturas escogidas, estaba formada por veinte docentes, casi todos con dedicación completa, aunque se incluyó a algún profesor asociado, por cuanto no se quería circunscribir el análisis exclusivamente a los profesores a tiempo completo y añadir la opinión de los que siguen vinculados a la profesión (aunque algunos profesores asociados tengan más experiencia docente que algunos noveles que han elegido la carrera académica exclusivamente).

En cuanto a los discentes, se buscó cierto grado de representatividad estadística en relación al curso en el que tienen matriculadas la mayor parte de materias, aunque añadiendo un factor corrector: se sobrerepresentó a los estudiantes de primero (a los que queda más tiempo de carrera y podrían aprovechar mejor el material si éste se implantara) en detrimento de los de

\footnotetext{
${ }^{3}$ Básicamente las referidas a redacción, locución, tecnología, lenguajes y géneros, así como algunas más concretas de cada licenciatura. No se trabajó con optativas porque suele haber menos alumnos. Por citar algunas: Redacción y Locución en Medios Audiovisuales; Teoría y Técnica del Lenguaje Radiofónico; Géneros Informativos y Rutinas de Producción en Radio y Televisión; Comunicación Corporativa; Géneros Radiofónicos; Entrevista y Reportaje Televisivos; Políticas de Comunicación; Propaganda y Publicidad Institucionales; y Tecnología de los Medios Audiovisuales.
} 
cuarto, que están ya en su recta final de licenciatura. Así, la muestra final (61 alumnos) estuvo formada por un $37,70 \%$ de estudiantes de primero, un $26,23 \%$ de segundo, un $22,95 \%$ de tercero y un $13,11 \%$ de cuarto. Finalmente, cabe añadir que las encuestas, tanto de docentes como de discentes, eran anónimas.

Una vez elaborada la animación y delimitada la muestra, se procedió a hacer pública la dirección de la página web básicamente a través de dos vías. En el caso de los discentes, se les comunicó personalmente en las clases y también se emitió un mensaje en el Campus Virtual, contando muy brevemente en qué consistía el estudio y quiénes eran los profesores implicados. En el caso de los docentes, se les envió un correo electrónico pero se aprovechó igualmente la cercanía y frecuencia de encuentro para recordárselo y animarles a participar.

Recordemos nuevamente, antes de abordar los principales resultados de las encuestas, que nos hallábamos en una primera fase del estudio y la animación elaborada no quedaba vinculada al desarrollo de ninguna asignatura concreta, no había formado parte del devenir de una materia durante un semestre $y$, aunque evidentemente sirvió a más de un alumno, no estaba pensada para ningún proceso de evaluación en concreto. En este primer estadio del análisis, sólo se pretendía interrogar a los principales implicados en el proceso de enseñanza-aprendizaje sobre su parecer con referencia a un material que bien podría formar parte en el futuro de dicho proceso. El objetivo final, pues, era obtener información que ayudara a mejorar el eventual diseño de nuevas animaciones.

\section{RESULTADOS}

Para contextualizar mejor los resultados, tanto docentes como discentes fueron consultados, en la primera parte de la encuesta, sobre cuestiones referidas al conocimiento y uso de algunas herramientas como el propio Camtasia Studio o Internet.

\section{Uso de la red y material docente de soporte}

Preguntados los profesores sobre si utilizaban material de soporte en sus clases, un $65 \%$ dijo que sí y un 35\% que no, los mismos porcentajes obtenidos al preguntar si habían producido ellos mismos algún tipo de material. Respecto al uso de Internet para preparar sus clases, los profesores respondieron tal y como se muestra en el gráfico 1 . 


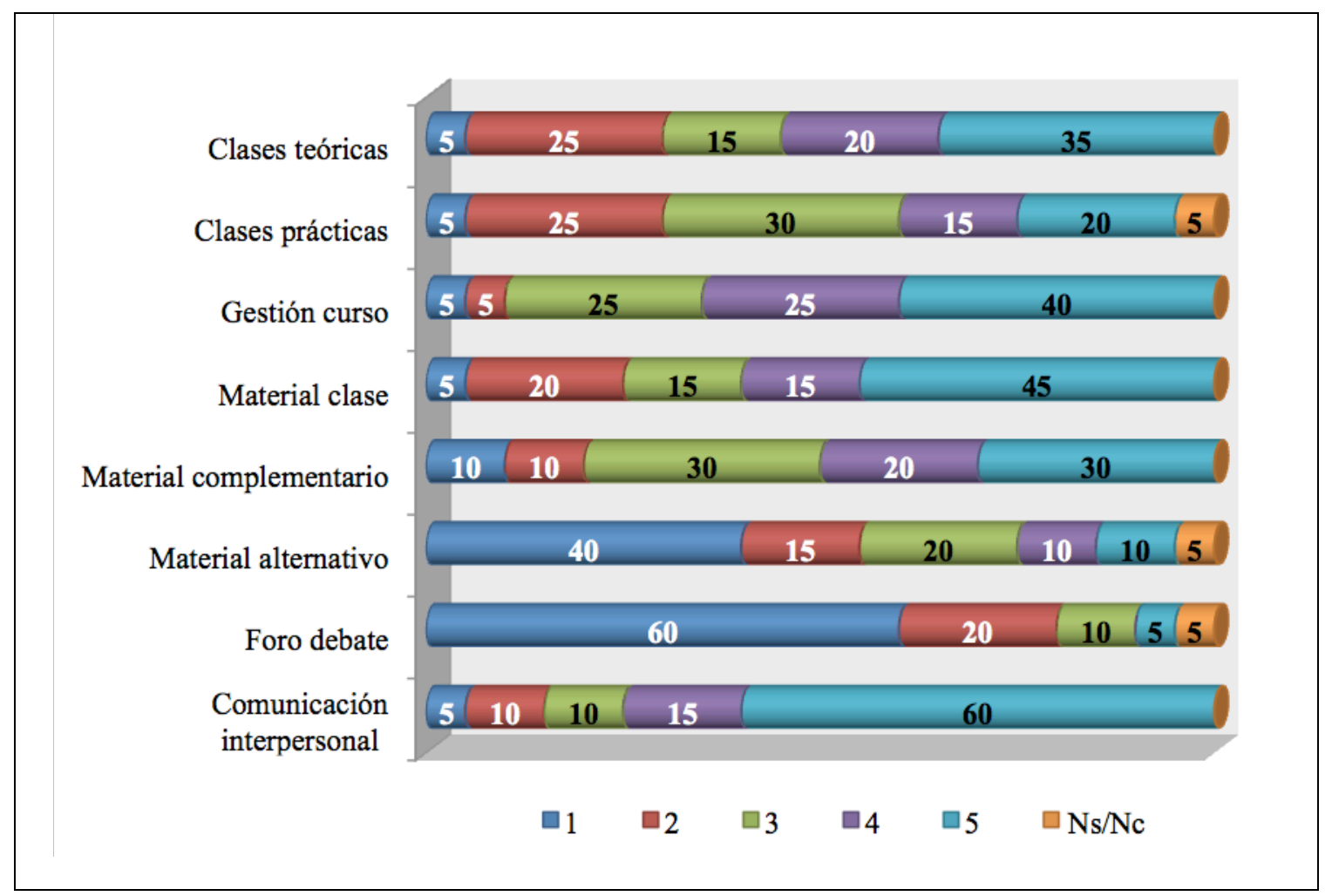

Gráfico 1. Uso de Internet para la preparación de las clases Fuente: elaboración propia. Nota: 1 (nada), 5 (mucho).

Entre los usos de Internet destacan mayoritariamente la gestión del propio curso y sus materiales, lo cual suele coincidir con el uso del Campus Virtual, plataforma institucional de la UAB que se puso a disposición de los profesores y alumnos por primera vez hace unos diez años. Por otro lado, su uso está mucho menos extendido como foro de debate o vía para proveer a los alumnos de material alternativo (material adicional del temario no visto en clase).

Por su parte, las respuestas de los alumnos guardan cierta proporción puesto que mayoritariamente utilizan la red para la gestión del curso: consultar notas, hacer trabajos y comunicarse con el docente. En mucha menor medida, usan Internet para buscar información de cuestiones no tratadas en clase, ampliar información del temario y para estudiar.

Cuando un profesor pone a disposición de sus alumnos material de soporte, la totalidad afirma utilizarlo. Los usos son distintos: el 88,5\% los emplea como refuerzo, el 55,7\% para profundizar en la materia, el $23 \%$ porque es obligatorio consultarlos, el $16,4 \%$ como sustituto de las clases y, finalmente, un $11,5 \%$ afirma darle otros usos.

Por otro lado, en cuanto a las preguntas referidas a la segunda parte de la encuesta, es decir, a las preguntas relacionadas directamente con la animación mostrada, destacan los siguientes resultados: 
Los docentes tienen una valoración muy similar respecto a la facilidad de uso de la animación visionada para uso propio y por parte de los alumnos. Un $55 \%$ de docentes considera que es una herramienta muy fácil para los alumnos y un $50 \%$, que lo es para uso propio. En el caso de los alumnos, sólo se les preguntó por la valoración para uso propio y aquí vale la pena destacar que los docentes habían considerado que resultaría más fácil de usar por los alumnos que ellos mismos. Casi todos se inclinaban por considerarlo muy fácil $(39,3 \%)$, pero hay más de quince puntos de diferencia respecto al $55 \%$ que les otorgaban los docentes.

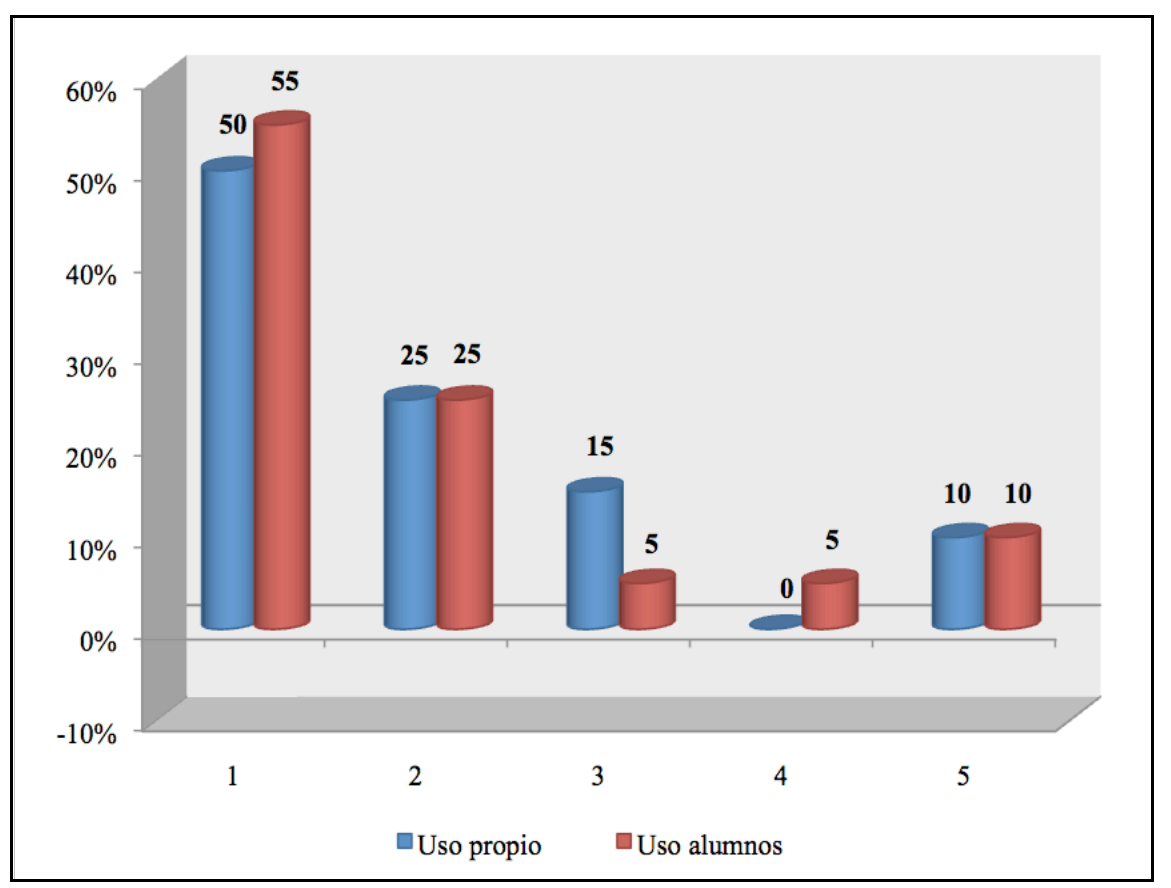

Gráfico 2. Valoración de la dificultad de uso de la animación (profesores) Fuente: elaboración propia.

Nota: 1 (muy fácil); 5 (muy difícil).

\section{Utilidad general de la animación}

Casi todos los encuestados encuentran alguna utilidad al material, aunque son los profesores los que parecen verle más salida para la docencia (45\%) que los alumnos para sus estudios $(27,8 \%)$. Sin embargo, más del $60 \%$ de éstos otorga a la animación las dos valoraciones más positivas (en una escala de 1 , nada útil, a 5 , muy útil).

Concretando un poco más, en el caso de los profesores, los componentes mejor valorados son el cuerpo de la letra, la duración y la información visual y los que menos, el dinamismo, el ritmo y la información sonora. 


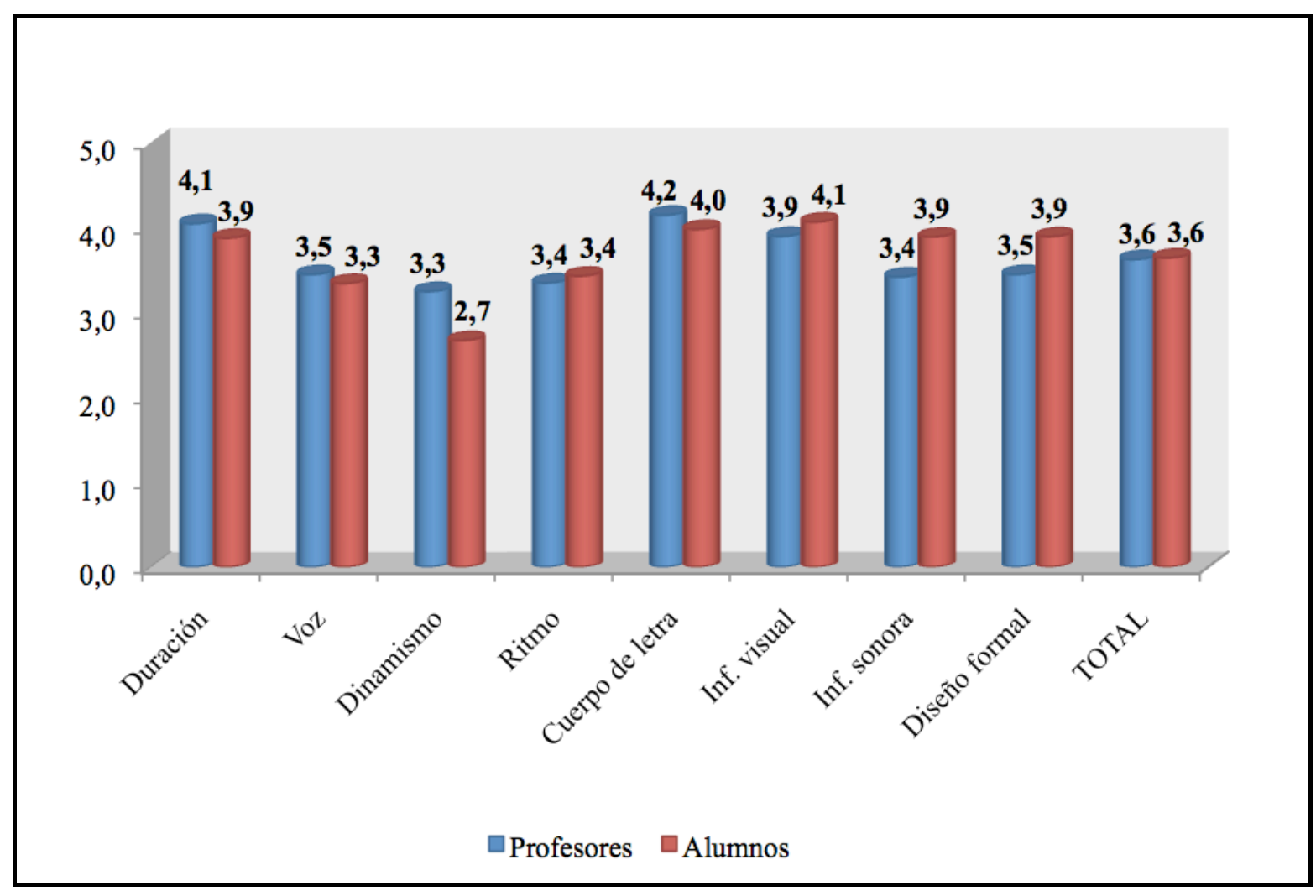

Grafico 3. Valoración de la utilidad de los componentes de la animación en la comprensión del contenido (profesores y alumnos)

Fuente: elaboración propia.

Nota: 1 (nada), 5 (mucho).

En el caso de los alumnos, lo más valorado es la información visual, el cuerpo de letra, la duración, la información sonora y el diseño formal (los tres últimos con la misma nota de 3,9). Entre lo menos valorado por los alumnos destaca el dinamismo, la voz y el ritmo.

Aunque lógicamente existen ciertas divergencias entra ambos colectivos, sin llegar a superar, sin embargo, las 6 décimas de diferencia, se observan tendencias claras para cada uno de los aspectos analizados: las valoraciones más positivas y las más negativas suelen coincidir en ambos casos. La nota media de las ocho variables es de 3,6 en ambos colectivos.

\section{Valoración de la cantidad de información de la animación}

Sin duda, uno de los elementos clave en toda la investigación es determinar las variables que sugieran un equilibrio en la muestra entre la cantidad justa de información y el tiempo de duración de la animación. Se trataba de encontrar un volumen medio sin obsesionarse pues, al ser interactiva, la persona que lo está visionando puede ir hacia adelante y hacia atrás tantas veces como desee. Tanto docentes como discentes le concedieron un valor medio, si bien no puede obviarse que un $41 \%$ de los alumnos y un $25 \%$ de los profesores creen que hay un exceso de información (valores 4 y 5). 


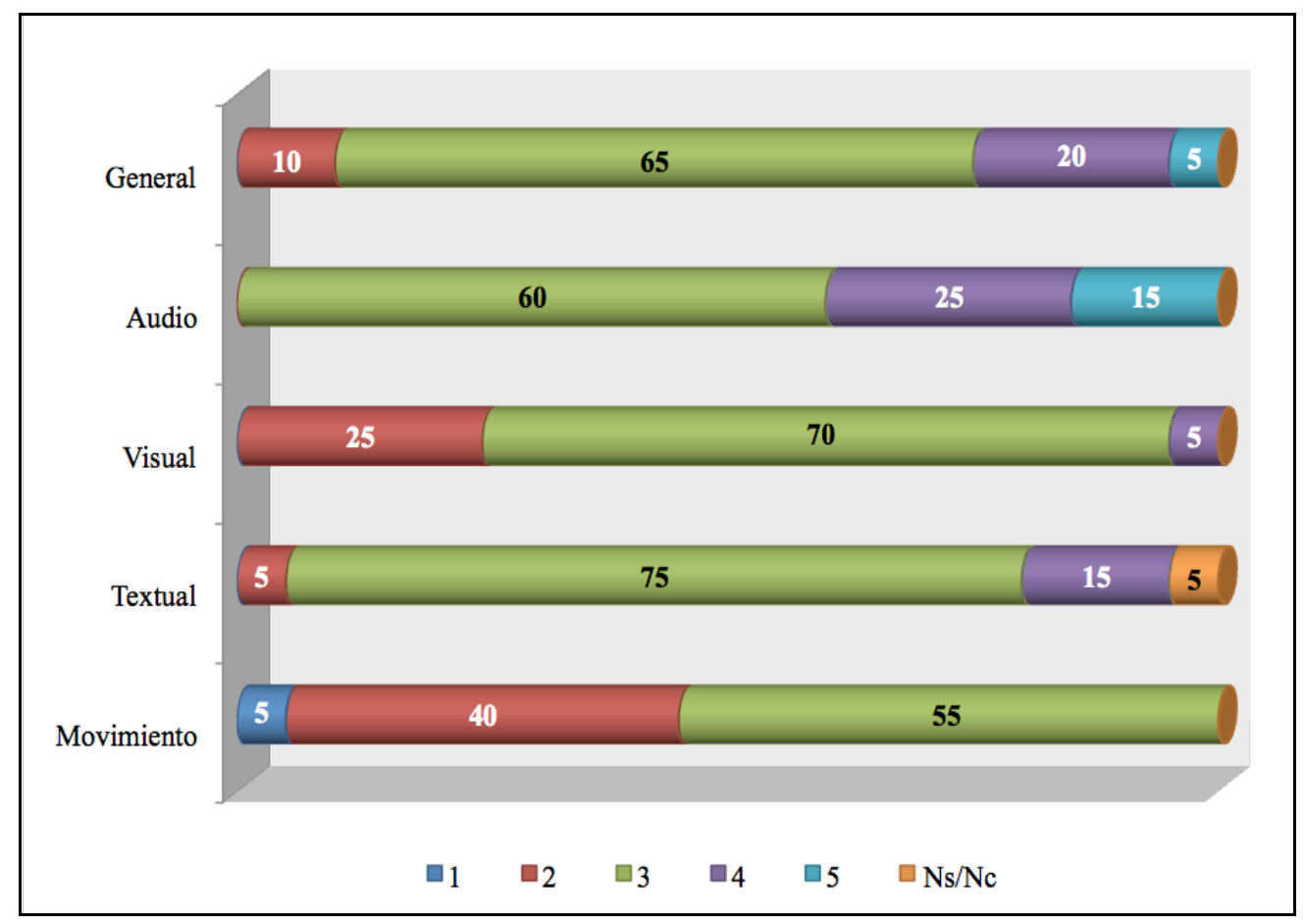

Gráfico 4. Valoración de la cantidad de información de la animación (profesores) Fuente: elaboración propia.

Nota: 1 (poca información); 5 (demasiada información)

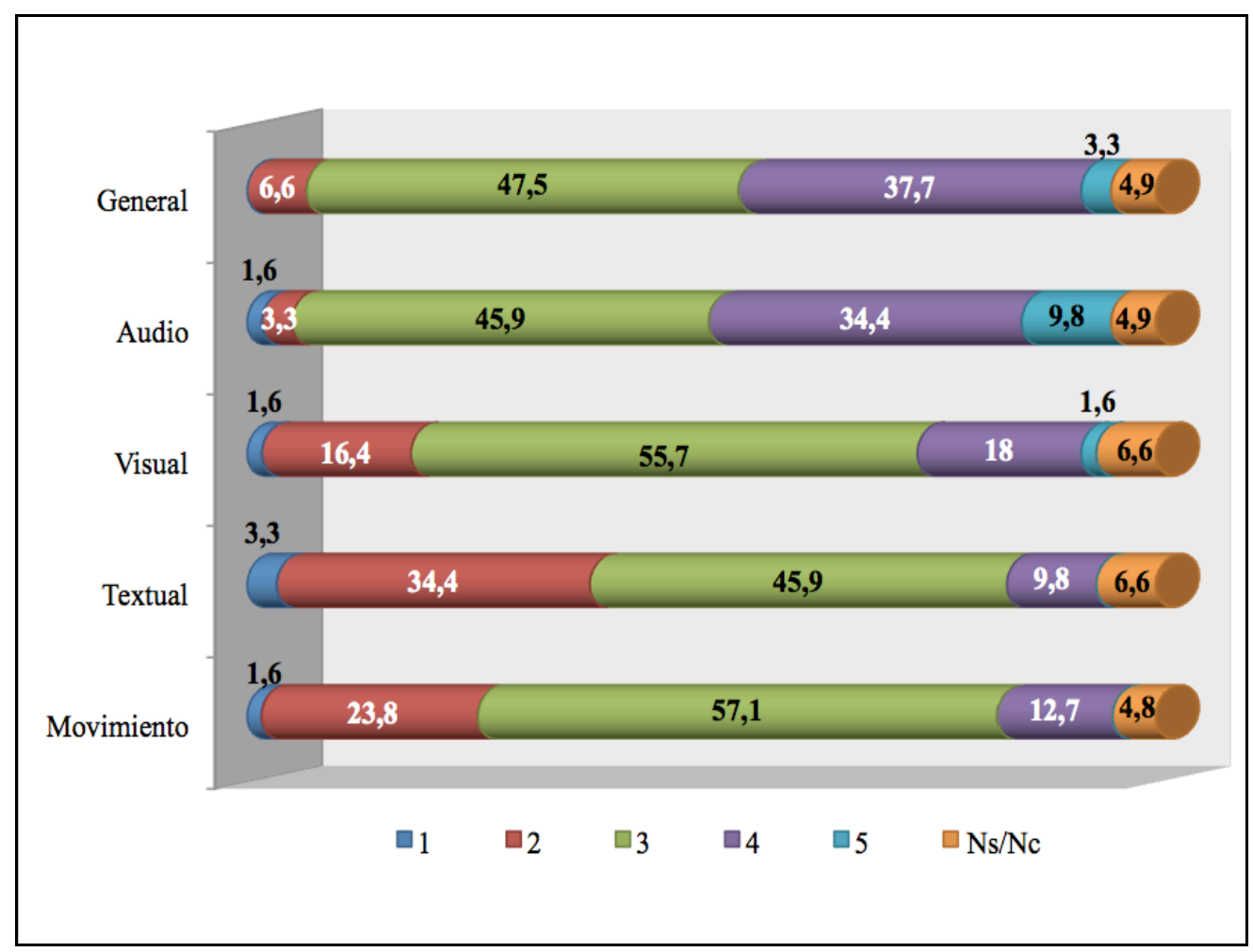

Gráfico 5. Valoración de la cantidad de información de la animación (alumnos)

Fuente: elaboración propia.

Nota: 1 (poca información); 5 (demasiada información) 
Los resultados de la pregunta referida a la cantidad de información deben servir de guía orientativa para la elaboración del protocolo de diseño y producción de material docente de soporte con Camtasia Studio.

Como puede observarse, el colectivo de profesores valora positivamente la cantidad de información contenida en el prototipo, especialmente en lo que se refiere al apartado textual y visual. Por su parte, los alumnos encuentran adecuada la cantidad de información y a su vez destacan positivamente los apartados movimiento y visual, justo los apartados en los que algunos profesores consideran que debería haber más cantidad. En resumen y en líneas generales, el colectivo de discentes, si tuviera que corregirse el prototipo, preferiría menos cantidad de información pero, a la vez, aumentaría la de tipo textual. Por su parte, los profesores reclamarían más movimiento y menos información audio.

Después, a los alumnos se les preguntó sobre la mayor o menor utilidad de este tipo de material docente respecto a otros y puede observarse en el gráfico 6 que equiparan la animación a los vídeos en línea $(55,7 \%)$ y la consideran más útil que los dossiers, los archivos sonoros y los documentos en línea, especialmente en el caso de los dos primeros (59\% en ambos).

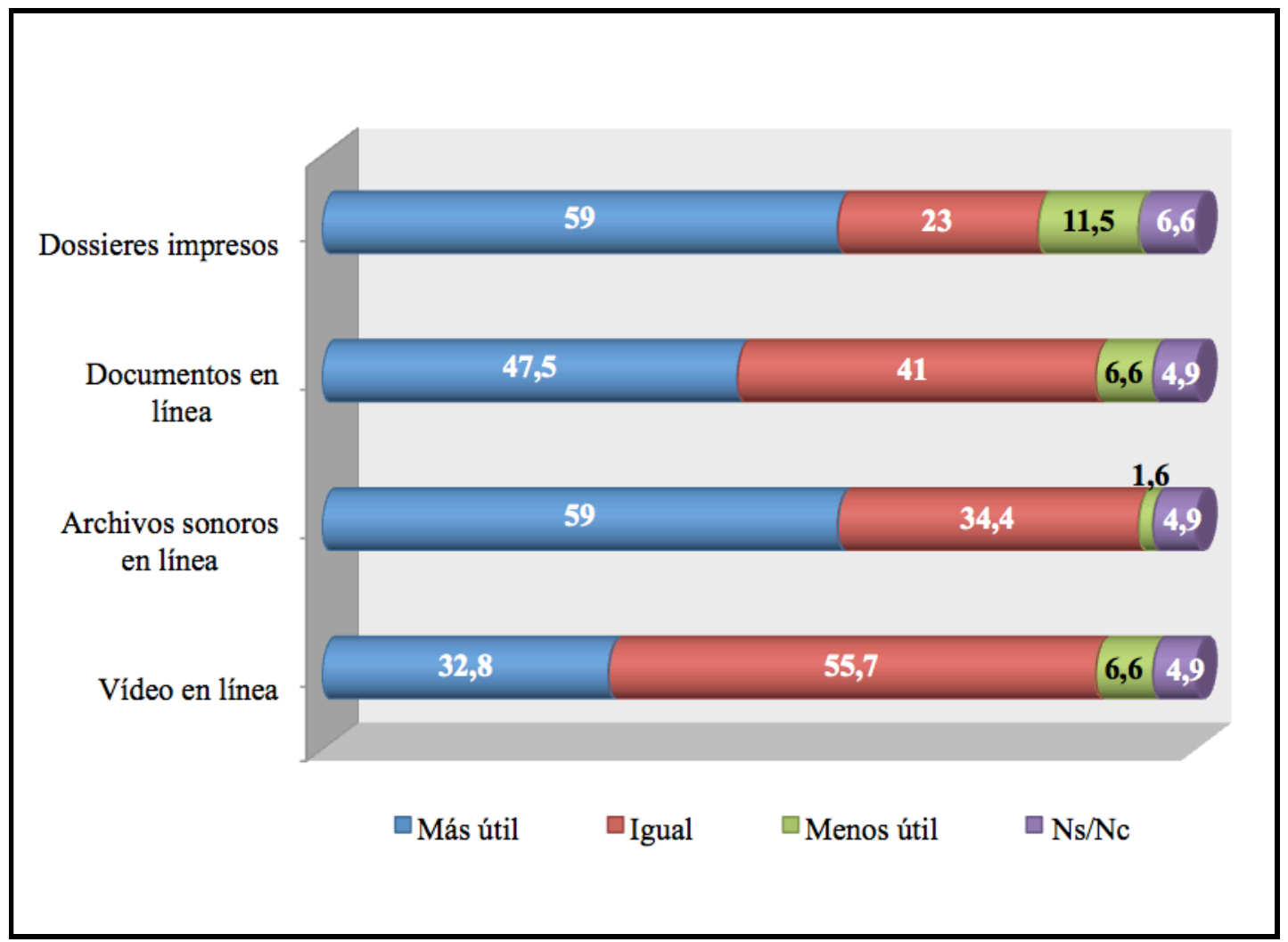

Gráfico 6. Utilidad de la animación respecto a otros recursos (alumnos)

Fuente: elaboración propia.

\section{Aplicaciones potenciales de la animación}

Las aplicaciones potenciales se refieren tanto a su utilidad para contenidos teóricos como prácticos. En el primer caso, se detecta una mayor percepción de utilidad entre los alumnos que entre los profesores ya que más del 75\% de discentes se inclina por las dos notas más altas, 4 y 5 (entre 1, nada útil, y 5 , muy útil) mientras que en el caso de los docentes sólo es el $55 \%$ quien las 
elige. Entre los profesores, el $20 \%$ se muestra escéptico sobre la aportación que podría hacer este tipo de material (mientras que entre los alumnos es sólo del $1,6 \%$ ). En lo que se refiere a su aplicación a las prácticas, la valoración desciende puesto que las notas más elevadas sólo las ha elegido el $40 \%$ de profesores y el $59 \%$ de alumnos.

\section{Impacto sobre el proceso de E-A}

La pregunta que se planteó era directa: ¿crees que el uso de esta herramienta cambiaría tu manera de estudiar / enseñar? Y lo más destacado es señalar que el $70 \%$ del profesorado cree que no cambiaría su método docente, mientras que el $45,9 \%$ de los alumnos responde que no cambiaría su forma de estudiar.

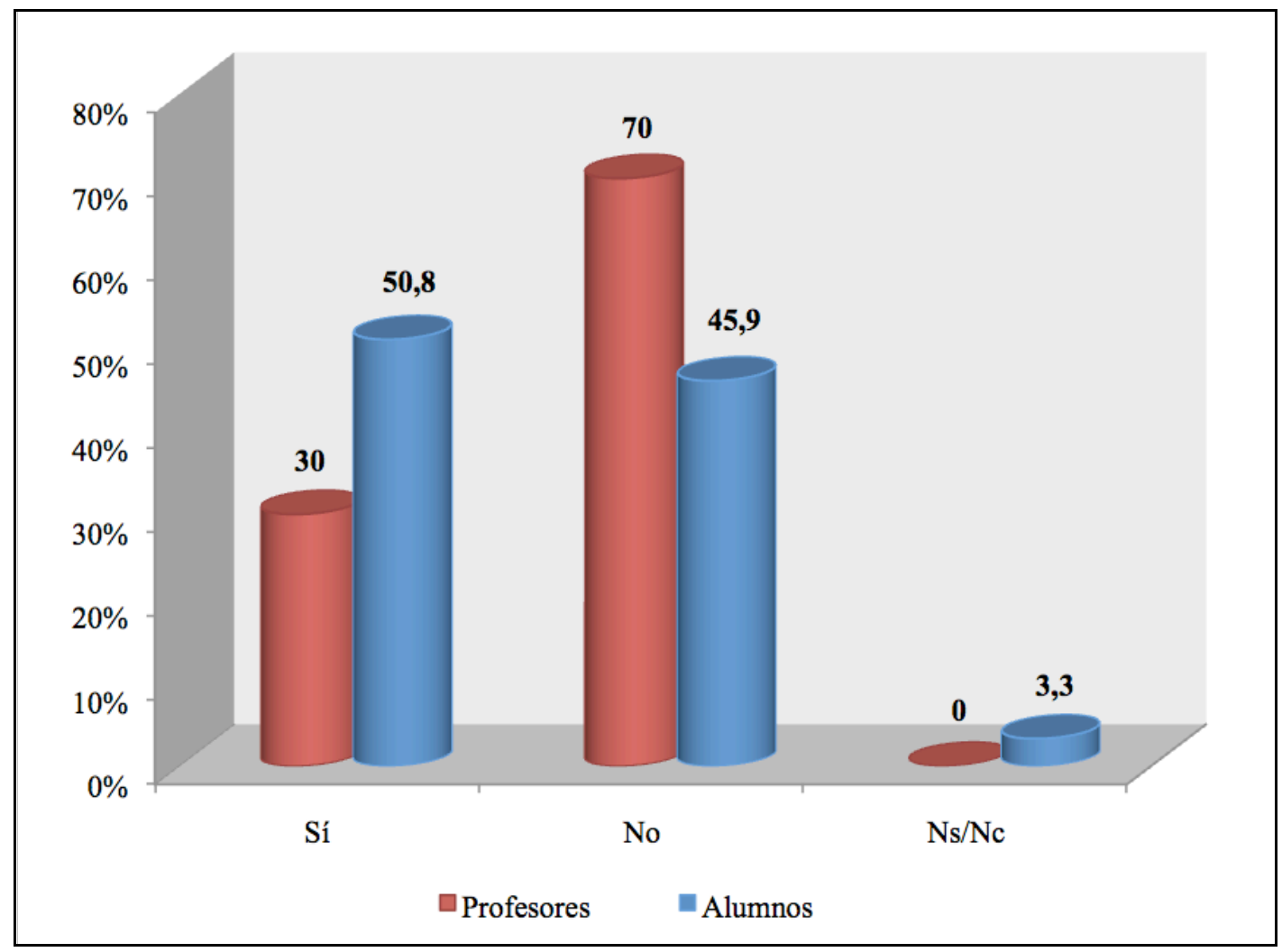

Gráfico 7. Cambios en el proceso de enseñanza-aprendizaje Fuente: elaboración propia.

Tanto a profesores como a alumnos se les pidió que anotasen hasta tres ejemplos de aplicación de este tipo de material en el proceso de enseñanza-aprendizaje. Resulta muy interesante señalar que respondieron menos de la mitad de los encuestados en ambos colectivos. Entre los profesores, se sugirió que podría utilizarse para estimular la interacción en el aula, para plantear problemas en clase a partir de su visionado, para reforzar la interacción entre teoría y práctica, para autoevaluación o para poder dedicar más tiempo a discutir en clase.

Los alumnos, por su parte, afirmaron que si utilizaran estos materiales cambiarían su forma de trabajar las asignaturas porque, como comentan muchos de ellos, les resultaría más fácil preparar los exámenes (afirman que el hecho de poder repetir la animación tantas veces como quieran es 
una clara ventaja ya que no se rompe el ritmo de clase y no es necesario preguntar constantemente al profesor) o podrían ampliar algunos aspectos de los apuntes tomados en clase.

Una de las afirmaciones más interesantes en este sentido es el hecho de poder atender más y mejor en clase a la explicación, sin preocuparse de tomar apuntes, pues luego podrían consultar la animación. En algunos casos, se sugiere tomarlo como sustituto de las clases aunque no sea un material diseñado para este fin. En realidad, resulta abrumadora la cantidad de respuestas referidas a la forma de tomar apuntes, su validez a la hora de estudiar y el propio proceso de estudio. Algunas de las respuestas apuestan incluso por su uso previo a la clase, de manera que se entienda mejor lo que se comenta. Uno de los alumnos apunta: "Las herramientas audiovisuales ayudan a retener más fácilmente la información así que supongo que recurriría todavía más a las técnicas mnemotécnicas: identificación de imágenes concretas con contenidos".

En resumen, de la lectura de todas las respuestas de los alumnos se desprende la idea de que aumentaría su productividad, resultaría más fácil la comprensión de la materia dentro y fuera del aula, sustituiría el "empollar" por el "aprender" de una manera más lúdica. Y, por supuesto, tienen muy claro que tendrían menos apuntes y los pocos que tuvieran serían de mejor calidad pues no se tomarían tanto al dictado sino que se basarían en conceptos, esquemas y atender más a la explicación del profesor.

\section{CONCLUSIONES}

Las experiencias en innovación docente a nivel superior, principalmente las de diseño de instrumentos pedagógicos, aplicadas a la formación de comunicadores son escasas en el ámbito universitario español, pese a la facilidad de acceso y uso que los discentes y profesores tienen de las nuevas tecnologías. En este sentido, la investigación realizada pretende que las nuevas tecnologías sean aprovechadas en pos de la formación profesional y, al mismo tiempo, impulsar un protocolo de producción de contenidos audiovisuales de soporte para la E-A.

En líneas generales, los resultados de las encuestas de esta primera fase del estudio ofrecen suficientes pistas como para poder afrontar con mayores garantías y seguridad la segunda parte. Además, las aparentes contradicciones surgidas a través de las respuestas dan mayor valor a la propuesta, ya planteada en el diseño inicial, de añadir otros instrumentos de investigación propios de las ciencias sociales como por ejemplo las entrevistas en profundidad. Ejemplos de tales contradicciones serían la percepción de la dificultad del uso de la animación o las preguntas referidas a su utilidad como soporte para contenidos teóricos y prácticos. Como se ha visto, los alumnos otorgan más valor que los profesores a su aplicación en las clases teóricas, lo cual no deja de ser curioso pues parecen olvidar la utilidad de los tutoriales que los mismos discentes reclaman a menudo. Puede que la naturaleza teórica del contenido elegido para elaborar la muestra haya podido introducir un sesgo en el resultado, posibilidad que se va a tener en cuenta en las siguientes fases del estudio.

Se ha podido comprobar también que los estudiantes utilizan el material de soporte alojado en los espacios virtuales de la red, sobre todo en los institucionales, para reforzar y profundizar los conocimientos adquiridos y facilitados por el profesor en el aula. En este sentido, existe una clara aceptación y tendencia al uso de material de soporte, lo que valida y justifica la idea de generar contenidos audiovisuales que se conviertan en facilitadores de acción pedagógica. 
Otro de los apartados en los que la realización de entrevistas en profundidad puede contribuir a entender mejor los datos obtenidos es el referido a posibles cambios en el proceso de enseñanzaaprendizaje. Como hemos visto, el $70 \%$ del profesorado cree que no cambiaría su método docente, mientras que el $45,9 \%$ de los alumnos no cambiaría su forma de estudiar. Podría resultar interesante preguntar a ambos colectivos el porqué de tal respuesta, teniendo en cuenta que en líneas generales se ha otorgado una buena puntuación a su uso como material de apoyo. ¿Puede mantenerse igual el método docente y la forma de estudiar cuando se incorporan materiales de este tipo? ¿Puede el proceso de enseñanza-aprendizaje mantenerse inamovible cuando se introduce en dicho proceso un elemento como el prototipo mostrado en la encuesta?

En líneas generales, las divergencias encontradas más que un déficit en el estudio se convierten en un activo a ponderar, es decir, las diferencias se advierten como una falta de costumbre $y$ consenso en el uso de esta clase de iniciativas de innovación docente por la casi inexistencia de este tipo de propuestas de educación a nivel superior, al menos en el ámbito académico observado. De todo lo dicho, se desprende la importancia ya no sólo de investigaciones que generen protocolos de producción de herramientas pedagógicas más complejas sino de su implementación en la dinámica de E-A a nivel superior.

\section{REFERENCIAS BIBLIOGRÁFICAS.}

Aiello, M. y Willem, C. (2004). El blended learning como práctica transformadora. Pixel-Bit, 23. Consultado el 4 de mayo de 2009, en http://www.sav.us.es/pixelbit/articulos/n23/n23art/art2302.htm.

Bonet, M. (2006). La centralidad de la comunicación audiovisual en el entorno digital: propuestas desde la experiencia formativa. RUSC, 3(2). Consultado el 1 de junio de 2009, en http://www.uoc.edu/rusc/3/2/dt/esp/bonet.pdf.

Bonet, M., Martí, J. M. y Pérez-Portabella, A. (2005). Assignatures experimentals: pràctica i creativitat. En J. Casal y A. Sastre (Eds.), Didàctica $i$ organització d'assignatures basades en l'experimentació. Barcelona: Universitat Politècnica de Catalunya.

Grané, M. (2004). Comunicación Audiovisual. Experiencia basada en el blended learning en la universidad. Pixel-Bit, 23. Consultado el 16 de mayo de 2009, en http://www.sav.us.es/pixelbit/articulos/n23/n23art/art2308.htm.

O'Day, D. H. (2006). How to Make Pedagogically Meaningful Animations for Teaching and Research Using PowerPoint ${ }^{\mathrm{TM}}$ \& Camtasia ${ }^{\mathrm{TM}}$. Consultado el 25 de mayo de 2009, en http://www.techsmith.com/community/education/research.asp\#animations.

Soriano, J. (2007). L'ofici de comunicòleg. Mètodes per investigar la comunicació. Vic: Eumo. 
RIBES, Xavier; BONET, Montse; GUIMERÀ, Josep A; FERNÁNDEZ, David; MARTíNEZ, Luísa (2009) «Multimedia e interactividad en el material docente de soporte y su aplicación a los estudios de comunicación» [artículo en línea]. EDUTEC, Revista Electrónica de Tecnología Educativa. Núm. 30 / Noviembre 2009. [Fecha de consulta: dd/mm/aa].

http://edutec.rediris.es/revelec2/revelec30/

ISSN 1135-9250. 


\section{ANEXO: Cuestionarios}

\subsection{Test previo a los alumnos}

\section{Datos de identificación}

1. NIU ${ }^{4}$

2. Sexo: Hombre / Mujer

3. Curso en el que estás matriculado (haz constar aquél en el que estés cursando la mayoría de asignaturas)

4. Licenciatura en la que estás matriculado

Comunicación Audiovisual / Periodismo / Publicidad y Relaciones Públicas

\section{Acceso a Internet y usos de la red}

5. ¿Tienes ordenador personal en casa?

6. ¿Tienes acceso a Internet en casa?

7. ¿Cuántos días de la semana accedes a Internet de media?

8. ¿Cuántas horas a la semana dedicas a navegar por Internet de media?

9. ¿A qué dedicas el tiempo de navegación por la red?

Entretenimento

Comunicación interpersonal (chat, correo)

Educación / formación

Consulta de noticias

Búsqueda de información sobre temas de tu interés

Compra

Otros

10. Cuando te conectas a Internet ¿sueles descargarte material?

Sonoro (1.- Nunca / 5.- Muy a menudo)

Audiovisual (1.- Nunca / 5.- Muy a menudo)

Fotográfico (1.- Nunca / 5.- Muy a menudo)

Textual (1.- Nunca / 5.- Muy a menudo)

\footnotetext{
${ }^{4}$ Es el Número de Identificación de Usuario, un número único asignado a cada miembro de la Universidad Autónoma de Barcelona.
} 
Software - programas - aplicaciones (1.- Nunca / 5.- Muy a menudo)

11. ¿Sueles utilizar Internet para tareas relacionadas con la carrera?

Puntúa de 1 (nada) hasta 5 (mucho)

Estudiar para los exámenes

Hacer las prácticas de las asignaturas

Hacer trabajos de fin de curso

Ampliar la información que ha dado el profesor

Buscar información de cuestiones no tratadas en clase

Buscar aclaraciones o información para resolver dudas

Consultar las notas

Comunicarme con el profesor

\section{Conocimiento y dominio de herramientas informáticas}

12. Valora el conocimiento y dominio que tienes de las siguientes herramientas informáticas puntuando entre 1 (nada) y 5 (mucho)

\begin{tabular}{|l|l|l|}
\hline & Conocimiento & Dominio \\
\hline Edición de textos & & \\
\hline Hojas de cálculo & & \\
\hline Bases de datos & & \\
\hline Presentaciones multimedia (Ej. PowerPoint) & & \\
\hline Edición de sonido & & \\
\hline Edición de video & & \\
\hline Edición web & & \\
\hline Aplicaciones de ilustración y compaginación & & \\
\hline Animación 2D o vectorial (Ej. Flash) & & \\
\hline Animación 3D & & \\
\hline Retoque fotográfico & & \\
\hline Grabación de sonido & & \\
\hline Grabación de video & & \\
\hline Fotografía & & \\
\hline
\end{tabular}




\section{$\underline{\text { Uso de material de soporte al estudio }}$}

13. ¿En cuántas asignaturas te has matriculado este curso?

14. ¿En cuántas de estas asignaturas el profesor ha puesto a tu disposición material de soporte docente en la red (web, blog, wiki, otros)?

15. ¿Utilizas el material que te ofrece el profesor? Sí / No

16. En caso afirmativo, ¿para qué lo usas?

Refuerzo de los contenidos recibidos en las clases

Profundización de lo explicado en clase

Como sustitutivo de las clases

Solamente cuando es obligatorio para superar la asignatura

Otros. Detalla, por favor, en qué casos los utilizas

17. En caso negativo, ¿’por qué no los utilizas?

Porque no es obligatorio para la asignatura

Porque el Campus Virtual es difícil de utilizar

Porque el material disponible no me resulta útil

18. ¿Podrías detallar brevemente en qué consiste la digitalización de los contenidos audiovisuales? 


\subsection{Test previo a los profesores}

\section{Datos de identificación}

1. NIU:

2. Sexo: Hombre / Mujer

3. Curso donde impartes clases este cuatrimestre

4. Asignaturas que impartes

5. Licenciaturas en las que impartes docencia este cuatrimestre

Comunicación Audiovisual / Periodismo / Publicidad y Relaciones Públicas

\section{Material de soporte y docencia}

6. ¿Has producido alguna vez material docente en soporte digital (Ej. PowerPoint, webs, blogs, podcast) para dejarlos a disposición de los alumnos?

7. ¿Usas este tipo de material digital de soporte, independientmente de que lo hayas elaborado tú o no?

8. ¿Has utilizado alguna vez material digital de soporte a la docencia en clase (PowerPoint, webs, blogs, etc.)?

9. ¿Sabes qué es el Camtasia Studio? Sí / No

10. En caso de haber respondido afirmativamente, ¿ipuedes describir sus características básicas?

11. ¿Lo has utilizado alguna vez en relación con la docencia?

12. Cuantifica el conocimiento y dominio que tienes de las siguientes herramientas informáticas puntuando entre 1 (nada) y 5 (mucho)

\begin{tabular}{|l|l|l|}
\hline & Conocimiento & Dominio \\
\hline Edición de textos & & \\
\hline Hojas de cálculo & & \\
\hline Bases de datos & & \\
\hline Presentaciones multimedia (Ej. PowerPoint) & & \\
\hline Edición de sonido & & \\
\hline Edición de video & & \\
\hline Edición web & & \\
\hline Aplicaciones de ilustración y compaginación & & \\
\hline Animación 2D o vectorial (Ej. Flash) & & \\
\hline Animación 3D & & \\
\hline Retoque fotográfico & & \\
\hline Grabación de sonido & & \\
\hline
\end{tabular}




\begin{tabular}{|l|l|l|}
\hline Grabación de video & & \\
\hline Fotografía & & \\
\hline
\end{tabular}

\section{$\underline{\text { Uso de la red en la docencia }}$}

13. ¿Sueles utilizar Internet en tareas relacionadas con la docencia?

Puntua de 1 (nada) a 5 (mucho) para:

Preparar las clases teóricas

Preparar las clases prácticas

Gestionar y organizar el curso (calendario de curso, publicar notas...)

Ofrecer a los estudiantes el material que doy en clase

Facilitar a los alumnos material complementario al ofrecido en el aula

Dar material que no daré en clase pero entra en el temario del curso

Organizar foros / debates con y entre los alumnos

Comunicarme con los alumnos

14. ¿Podrías detallar brevemente en qué consiste la digitalitzación de los contenidos audiovisuales? Sí / No 


\subsection{Test para los alumnos tras la visualización}

\section{Usabilidad y características de la animación}

1. ¿Has tenido algún problema técnico en la reproducción de la animación?

2. En caso afirmativo, ¿crees que ha afectado a la comprensión del contenido? Sí / No

3. Valora la facilidad de uso de la animación (1.- Muy fácil / 5.- Muy difícil)

4. ¿Cuántas veces la has reproducido?

\section{$\underline{\text { Utilidad general de la herramienta }}$}

5. ¿Crees, en general, que este tipo de material docente es una herramienta útil para tus estudios?

(1.- Nada / 5.- Mucho)

6. Valora la utilidad de cada componente de la animación en la comprensión del contenido:

(1.- Nada / 5.- Mucho)

Duración

Voz

Dinamismo (variación del contenido)

Ritmo de la animación

Cuerpo de letra

Información visual

Información sonora

Diseño formal de la animación (dimensiones, botones de navegación, etc.)

7. Valora la cantidad de información que contiene esta cápsula. 1.- Poco / 5.- Demasiado

8. Valora la cantidad de información que aporta el audio. 1.- Poco / 5.- Demasiado

9. Valora la cantidad de información visual de esta cápsula. 1.- Poco / 5.- Demasiado

10. Valora la cantidad de información textual de esta cápsula. 1.- Poco / 5.- Demasiado

11. Valora la cantidad de movimento de esta cápsula. 1.- Poco / 5.- Demasiado

12. Comparado con otros recursos de soporte a la docencia, éste que has visionado crees que es...

[Las opciones de respuesta son: “Más útil que / Igual de útil que / Menos útil que”]

Dossier impreso

Documentos en línea

Archivos sonoros en línea 
Vídeos en línea

\section{$\underline{\text { Aplicaciones potenciales de la herramienta }}$}

13. Valora la utilidad que puede tener este tipo de material de soporte en la explicación de contenidos teóricos y prácticos

Teóricos (1.- Nada útil / 5.- Muy útil)

Prácticos (1.- Nada útil / 5.- Muy útil)

14. Cita cinco asignaturas donde creas que este tipo de material sería especialmente útil. Ordénalas de mayor a menor utilidad

15. Cita hasta tres ejemplos de contenidos de asignaturas elaborados en la línea del que has visto y que te gustaría tener a tu disposición

\section{$\underline{\text { Depósito de recursos en línea }}$}

16. ¿Te gustaría que existiera un depósito digital donde encontrar animaciones de este tipo, relacionadas con todas o buena parte de las asignaturas que cursas?

17. Este depósito debería ser...

Público, abierto a todos los alumnos y profesores

Limitado, con acceso solamente para profesores que nos los facilitarían de acuerdo a las necesidades de la asignatura

18. Si existiera este depósito, ¿uutilizarías el material disponible como soporte para el estudio?

\section{$\underline{\text { Impacto sobre la docencia }}$}

19. ¿Crees que el uso de esta herramienta cambiaría tu forma de estudiar? Sí / No

20. En caso afirmativo, cita hasta tres aspectos concretos en que crees que cambiaría tu manera de preparar las asignaturas 


\subsection{Test para los profesores tras la visualización}

\section{Usabilidad y características de la animación}

1. ¿Has tenido algún problema técnico en la reproducción de la animación?

2. En caso afirmativo, ¿crees que ha afectado a la comprensión del contenido? Sí / No

3. Valora la facilidad de uso de la animación desde tu punto de vista como docente

1.- Muy fácil / 5.- Muy difícil

4. Valora si la animación es facil de usar para los alumnos

1.- Muy fácil / 5.- Muy difícil

\section{Utilidad general de la herramienta}

5. ¿Crees, en general, que los materiales producidos con Camtasia pueden ser útiles para la docencia?

$$
\text { (1.- Nada / 5.- Mucho) }
$$

6. Valora la utilidad de cada componente de la animación en la comprensión del contenido:

(1.- Nada / 5.- Mucho)

Duración

Voz

Dinamismo (Variación del contenido)

Ritmo de la animación

Cuerpo de letra

Información visual

Información sonora

Diseño formal de la animación (dimensiones, botones de navegación, etc.)

7. Valora la cantidad de información que contiene esta cápsula 1.- Poco / 5.- Demasiado

8. Valora la cantidad de informació que aporta el audio. 1.- Poco / 5.- Demasiado

9. Valora la cantidad de información visual de esta cápsula. 1.- Poco / 5.- Demasiado

10. Valora la cantidad de información textual de esta cápsula. 1.- Poco / 5.- Demasiado

11. Valora la cantidad de movimento de esta cápsula. 1.- Poco / 5.- Demasiado

\section{Aplicaciones potenciales de la herramienta}

12. Valora la utilidad que puede tener este tipo de material de soporte en la explicación de contenidos teóricos y prácticos

Teóricos (1.- Nada útil / 5.- Muy útil) 
Prácticos (1.- Nada útil / 5.- Muy útil)

13. ¿Crees que se podría utilizar en tu/s asignatura/s? Sí / No

14. Cita tres asignaturas, que no sean las tuyas, donde crees que esta herramienta podría ser especialmente útil. Ordenalas de mayor a menor utilidad

15. Cita, hasta un máximo de tres, ejemplos concretos de contenidos que tú ofrecerías a los alumnos en caso de poder utilizar Camtasia Studio

\section{Producción de contenidos y depósito de recursos en línea}

16. Si existiera un protocolo para el uso de Camtasia Studio,

a) ¿te animarías a producir contenidos?

b) ¿preferirías que hubiera alguien que los produjera a partir de tus directrices (esto implicaría conocer los rudimentos básicos de la herramienta)? Sí / No

c) No creo que me animara

17. ¿Te gustaría que existiera un depósito digital donde encontrar animaciones de este tipo para utilizar en la docencia?

18. En caso afirmativo, este depósito crees que debería ser...

Público, abierto a todos los alumnos y profesores

Limitado, con acceso solamente para profesores

19. Si existiera este depósito, ¿utilizarías el material disponible en tus asignaturas? Sí / No

\section{$\underline{\text { Impacto sobre la docencia }}$}

20. ¿Crees que el uso de esta herramienta cambiaría tu forma de hacer docencia? Sí / No

21. En caso afirmativo, cita hasta tres aspectos concretos en que crees que tu método docente podría cambiar 